\title{
Article
}

\section{Healthcare providers' perspectives on perceived barriers and facilitators of compassion: Results from a grounded theory study}

Singh, Pavneet, Raffin-Bouchal, Shelley, McClement, Susan, Hack, Tom, Stajduhar, Kelli, Hagen, Neil A, Sinnarajah, Aynharan, Chochinov, Harvey Max and Sinclair, Shane

Available at http://clok.uclan.ac.uk/22107/

Singh, Pavneet, Raffin-Bouchal, Shelley, McClement, Susan, Hack, Tom, Stajduhar, Kelli, Hagen, Neil A, Sinnarajah, Aynharan, Chochinov, Harvey Max and Sinclair, Shane (2018) Healthcare providers' perspectives on perceived barriers and facilitators of compassion: Results from a grounded theory study. Journal of Clinical Nursing . ISSN 0962-1067

It is advisable to refer to the publisher's version if you intend to cite from the work. http://dx.doi.org/10.1111/jocn.14357

For more information about UCLan's research in this area go to http://www.uclan.ac.uk/researchgroups/ and search for <name of research Group>.

For information about Research generally at UCLan please go to http://www.uclan.ac.uk/research/

All outputs in CLoK are protected by Intellectual Property Rights law, including Copyright law. Copyright, IPR and Moral Rights for the works on this site are retained by the individual authors and/or other copyright owners. Terms and conditions for use of this material are defined in the policies page. 
DR. PAVNEET SINGH (Orcid ID : 0000-0002-5865-669X)

Article type : Original Article

Healthcare providers' perspectives on perceived barriers and facilitators of

compassion: Results from a grounded theory study

Running title: Barriers and facilitators of compassion

Pavneet Singh $^{1}$, Shelley Raffin-Bouchal ${ }^{1}$, Susan McClement ${ }^{2,3}$, Thomas F. Hack ${ }^{2,3,4}$, Kelli

Stajduhar $^{5}$, Neil A. Hagen ${ }^{6,7}$, Aynharan Sinnarajah ${ }^{6,8}$, Harvey Max Chochinov ${ }^{2,9}$, and Shane Sinclair ${ }^{1,6}$

${ }^{1}$ Faculty of Nursing, University of Calgary, 2500 University Drive NW Calgary, Alberta Canada. T2N 1N4.

${ }^{2}$ Research Institute in Oncology Hematology, Cancer Care Manitoba, 4005E - 675

McDermot, Winnipeg, Manitoba, Canada. R3E 0V9.

${ }^{3}$ College of Nursing, Rady Faculty of Health Sciences, University of Manitoba, 89 Curry

Place, Winnipeg, Manitoba, Canada. R3T 2N2.

${ }^{4}$ School of Health Sciences, University of Central Lancashire, Brook Building $\left(4^{\text {th }}\right.$ Floor,

Room BB440), Preston, United Kingdom PR1 2HE.

${ }^{5}$ School of Nursing and Institute on Aging and Lifelong Health, University of Victoria, R

Hut, Room 103, Victoria, British Columbia, Canada. V8P 5C2.

${ }^{6}$ Department of Oncology, Cumming School of Medicine, University of Calgary, 2500

University Drive NW, Calgary, Alberta, Canada. T2N 1N4.

This article has been accepted for publication and undergone full peer review but has not been through the copyediting, typesetting, pagination and proofreading process, which may lead to differences between this version and the Version of Record. Please cite this article as doi: $10.1111 /$ jocn. 14357

This article is protected by copyright. All rights reserved. 
${ }^{7}$ Departments of Clinical Neurosciences and Medicine, Cumming School of Medicine, University of Calgary, 2500 University Drive NW, Calgary, Alberta, Canada. T2N 1N4.

${ }^{8}$ Palliative / End of Life Care, Calgary Zone, Alberta Health Services, 710 South Tower, $1403-29^{\text {th }}$ Street NW, Calgary, Alberta, Canada. T2N 2T8

${ }^{2}$ Department of Psychiatry, University of Manitoba, 771 Bannatyne Avenue, Winnipeg, Manitoba, Canada. R3E 3N4.

Corresponding Author: Shane Sinclair, sinclair@ucalgary.ca, +1(403) 220-2925

Acknowledgements: We would like to acknowledge and thank the research participants for their willingness to share their knowledge and experience. We also wish to thank the University of Calgary's University Relations for their expertise in designing the HCP Compassion model that is adapted herein to depict facilitators, challenges, adaptive and maladaptive responses to compassion. Competing Interest Statement: The authors declare no conflicts of interest.

Funding Statement: This study was supported by a MSI Foundation Grant (Grant \#880).

\title{
Healthcare providers' perspectives on perceived barriers and facilitators of compassion: Results from a grounded theory study
}

\begin{abstract}
Background: Compassion is considered a component of quality healthcare that healthcare providers (HCPs) are increasingly expected to provide. While there have been some studies exploring facets of HCPs' perspectives on the barriers and facilitators to providing compassion, a comprehensive understanding based on direct reports from HCPs is lacking. Objective: The aim of this study was to explore HCP perspectives and experiences of perceived barriers and facilitators of compassion.
\end{abstract}

This article is protected by copyright. All rights reserved. 
Methods: This study used Straussian grounded theory to examine HCP perspectives. Semistructured focus groups with frontline HCPs and individual interviews with peer-nominated exemplary compassionate care providers were audio-recorded, professionally transcribed, and analyzed. Fifty-seven participants were recruited from 3 healthcare settings within both rural and urban settings in Alberta, Canada, using convenience, snowball, and theoretical sampling.

Results: Qualitative analysis of the data generated two categories and associated themes and sub-themes delineating perceived barriers and facilitators to compassion. The first category, challenges to compassion, reflects participants' discomfort associating the notion of barriers to compassion, and contained several themes participants conceptualized as challenges: personal challenges, relational challenges, systemic challenges, and maladaptive responses. The second category, facilitators of compassion, included the themes of: personal facilitators, relational facilitators, systemic facilitators, and adaptive responses of intentional action.

Conclusion: Although participants described certain factors such as system and time constraints along with interaction styles of patients and families that can challenge HCP compassion, these challenges were not considered insurmountable. While acknowledging these as challenges, participants identified HCPs themselves, including their responses towards the identified challenges of compassion, as significant factors in this process - $\mathrm{a}$ novel finding from this study. This study provides insight into HCPs' perspectives on the notion of barriers and facilitators in the provision of compassion.

Relevance to clinical practice: This study provides a blueprint for optimizing compassion on a personal, relational and system level.

Keywords: Compassion, compassionate care, facilitators, challenges, barriers, healthcare providers, grounded theory, qualitative

This article is protected by copyright. All rights reserved. 


\section{INTRODUCTION}

Compassion is considered essential in providing quality healthcare, with compassion being described as the foundation of person-centered care (Frampton, Guastello, \& Lepore, 2013). Compassion in healthcare has been defined as "a virtuous response that seeks to address the suffering and needs of a person through relational understanding and action" (S. Sinclair, McClement, et al., 2016). Many healthcare providers (HCPs) feel drawn to the healthcare field because they want to provide compassionate care. While all patients expect their physicians, nurses and HCPs to be compassionate, what is known about providing compassion in these deeply challenging environments? Previous studies involving HCPs have focused on specific factors which could facilitate or inhibit compassionate care in healthcare, but these studies either did not identify the perspectives of HCPs directly or did not describe the barriers or facilitators in a comprehensive manner. The aim of this study was therefore to gain direct and practical insight into the perceived barriers and facilitators of compassion from those individuals directly delivering compassion-HCPs.

Three foundational domains of compassion barriers and facilitators can be distilled from this disparate literature: personal (Barratt, 2017; Brown, Crawford, Gilbert, Gilbert, \& Gale, 2014; Gale, Schroder, \& Gilbert, 2017; Hem \& Heggen, 2004; Jones, Winch, Strube, Mitchell, \& Henderson, 2016; Roberts, Warner, Moutier, Geppert, \& Green Hammond, 2011; Van Der Cingel, 2011), workplace/systems (Barron, Sloan, \& Deery, 2017; Bray, O'Brien, Kirton, Zubairu, \& Christiansen, 2014; Brown et al., 2014; Curtis, Horton, \& Smith, 2012; Dewar \& Mackay, 2010; Dewar \& Nolan, 2013; Fernando \& Consedine, 2017; Horsburgh \& Ross, 2013; Jones et al., 2016; Lown, Rosen, \& Marttila, 2011; Nolan, 2016; Smith,

Gentleman, Loads, \& Pullin, 2014), and relational barriers between HCPs and their patients (A. T. Fernando \& N. S. Consedine, 2014; Jones et al., 2016; Vivino, Thompson, Hill, \& Ladany, 2009). Insights into the personal domain is currently under developed in comparison

This article is protected by copyright. All rights reserved. 
to the relational and workplace/systems domains. The research to date on personal facilitators of compassionate care among HCPs identified previous personal health issues (Roberts et al., 2011), personal values (Jones et al., 2016), exposure to patient suffering (Van Der Cingel, 2011), contemplative practices and practicing self-care (Barratt, 2017; Gale et al., 2017) as important facilitators. Conversely, personal frustrations, HCP family issues, and feeling incompetent, disregard, disrespect, or hostility toward patients were identified as personal barriers to compassion (Brown et al., 2014; Hem \& Heggen, 2004).

In contrast, facilitators and barriers of compassion related to the workplace/system have received extensive attention and as a result are often considered to be the chief factor in facilitating or inhibiting HCPs' ability to deliver compassionate care (Barron et al., 2017; Bray et al., 2014; Brown et al., 2014; Curtis et al., 2012; Dewar \& Mackay, 2010; Dewar \& Nolan, 2013; Fernando \& Consedine, 2017; Horsburgh \& Ross, 2013; Jones et al., 2016; Lown et al., 2011; Nolan, 2016; Smith et al., 2014). In particular, a positive workplace, where providers felt supported by their employers and from their coworkers was felt to facilitate compassionate care (Barron et al., 2017; Dewar \& Mackay, 2010; Dewar \& Nolan, 2013; Jones et al., 2016). In contrast, work environments that were described as stressful, busy, negative, and resistant to change were identified as inhibiting compassion (Curtis et al., 2012; Horsburgh \& Ross, 2013). Other factors impacting HCPs' ability to provide compassionate care within the workplace/culture of care domain identified in the literature include competing professional demands, limited resources, and a lack of time (Barron et al., 2017; Bray et al., 2014; Brown et al., 2014; Curtis et al., 2012; Horsburgh \& Ross, 2013; Lown et al., 2011; Nolan, 2016; Smith et al., 2014). In addition, economic constraints, overly target/outcome driven healthcare systems and teaching environments focused primarily on knowledge-based competencies, with little value or dedicated time focused on cultivating skills and practices related to compassion were also identified as barriers (Bray et al., 2014;

This article is protected by copyright. All rights reserved. 
Smith et al., 2014).

Additional workplace/system factors identified specifically by physicians were medical specialty and professional experience. One study described that general practitioners and Internal Medicine specialists, along with less experienced physicians, had more barriers to compassion compared to psychiatrists and more experienced doctors (Fernando \& Consedine, 2017).

Finally, a few studies identified relational barriers and facilitators between HCPs and patients in the provision of compassionate care. Opportunities to develop positive relationships with patients and families; exposure to patient suffering; and patients who were perceived as self-motivated, likable, and articulate were considered relational facilitators to compassion (Jones et al., 2016; Vivino et al., 2009). Patient related relational barriers perceived by HCPs were patients who were resistant, aggressive, violating boundaries or difficult (A. T. Fernando \& N. S. Consedine, 2014; Vivino et al., 2009). Conversely, HCP relational barriers were identified as contempt toward patients, disregard, negativity, and having a pre-existing poor clinical relationship with a patient (A. T. Fernando \& N. S. Consedine, 2014; Vivino et al., 2009).

While this synthesis of the literature provides insight into a wide range of facets and apparent domains associated with barriers and facilitators of compassion, we could find no studies that comprehensively mapped them, including their relationship with one another, from the perspective of HCPs. Importantly, due to the lack of HCP accounts on the topic as a whole, there is little certainty as to whether the proposed facilitators and barriers of compassion identified by researchers are congruent with HCPs themselves. To address this gap, we conducted a grounded theory study exploring HCPs' perspectives on the nature of compassion and the associated facilitators and barriers within the context of healthcare specifically. The former study aim generated a HCP model of the construct of compassion 
which has been previously reported (Shane Sinclair et al., 2018), wherein participants were asked to define compassion which led to compassion being conceptualized as "a virtuous and intentional response to know a person, to discern their needs and ameliorate their suffering through relational understanding and action". On the other hand, this study aimed to gain insight into the perceived barriers and facilitators of compassion from the perspective of HCPs.

\section{METHODS}

\section{Study population}

Using convenience, snowball, and theoretical sampling, 57 HCPs were recruited from the palliative care services of the Calgary Zone in Alberta, Canada, between October 2015 and September 2016. The palliative care services spanned urban and rural settings and included hospice, homecare, hospital based consult teams, and a dedicated inpatient palliative care unit. The research protocol was approved by the University of Calgary Conjoint Health Research Ethics Board (\#REB 15-1999). Participants were eligible for the study if they were at least 18 years of age, able to speak and read English, able to provide written informed consent, and had worked in palliative care for a minimum of six months. While we recognize that compassion is an essential component of any healthcare setting, we selected participants from palliative care because compassion is of great significance in a healthcare environment which provides care where suffering is prevalent. To recruit participants, an overview of the study was presented at clinical care team meetings. A final sample size of 57 participants was required to achieve data saturation. The demographic characteristics of the participants are described in Table 1.

This article is protected by copyright. All rights reserved. 


\section{Data collection}

Data were collected from the participants using focus groups and one-on-one semistructured interviews. An interview guide (Table 2) was used for the interviews and was developed based on our literature reviews (S. Sinclair, Kondejewski, Raffin-Bouchal, KingShier, \& Singh, 2017; S. Sinclair, Norris, et al., 2016; S. Sinclair, Raffin-Bouchal, Venturato, Mijovic-Kondejewski, \& Smith-MacDonald, 2017; S. Sinclair, Russell, Hack, Kondejewski, \& Sawatzky, 2016) and the research team's previous experience in conducting qualitative studies (S. Sinclair, Beamer, et al., 2017; S. Sinclair, Bouchal, Chochinov, Hagen, \& McClement, 2012; S. Sinclair, McClement, et al., 2016; S. Sinclair, Torres, et al., 2016). While the guiding questions were designed to facilitate conversations, including potential barriers and facilitators, two targeted questions were developed to address this topic specifically: What do you feel are the major influencers of compassionate care in your practice? And what do you feel inhibits your ability to provide compassionate care? The latter question was initially worded to address the topic of compassion fatigue specifically due to the prominence of this phenomena within the healthcare literature (Figley, 1995; Mathieu, 2007; S. Sinclair, Raffin-Bouchal, et al., 2017), however we modified it to its current form as we felt in its original form it was a leading and presumptuous question. The interview guide also was modified slightly at each stage of data collection (Table 3, Table 4) based on the data which emerged in the earlier stages of this study, which is congruent with the concept of theoretical sensitivity and the iterative nature of grounded theory (Glaser \& Strauss, 1967; Strauss \& Corbin, 1990), where data-collection, data analysis, and theory generation occur simultaneously. Data collection in this study consisted of three stages. The first stage was comprised of 7 focus groups with 35 frontline HCPs. The aim of these focus groups was to obtain an understanding of compassion from the perspective of a broad spectrum of HCPs involved in direct patient care. Stage 1 HCPs were also asked to nominate two of their peers 
whom they considered exemplary compassionate care providers. In the second stage, 15 oneon-one interviews were conducted with the nominated HCPs to gain a deeper understanding of the qualities, skills, motivators, knowledge, behaviours, barriers and facilitators of compassion. Identified peers were informed about their nomination via their work email, in a sequential rank-ordered fashion, beginning with the individual with the most nominations. Nominated peers were asked to contact the study research coordinator if they were interested in participating. Fifteen nominees needed to be approached to reach our target sample of fifteen, amounting to a $100 \%$ response rate, with three of these participants having also participated in Stage 1 focus groups. The final stage of data collection was implemented at the end of the study, after all data from Stage 1 and 2 had been analyzed and an initial theoretical model had been developed. The final stage involved two focus groups with 5 study participants and 10 key stakeholders (administrators, clinical leads and healthcare faculty educators) in order to assess the validity of the results, and facilitate knowledge translation and future research directions. With the exception of the first two focus groups in Stage 1 and Stage 3, which were conducted by the Principal Investigator, all interviews and focus groups were conducted by an experienced qualitative interviewer previously unknown to participants. A detailed account of the data collection associated with this study were published in a separate manuscript (Shane Sinclair et al., 2018).

\section{Data Analysis}

Straussian grounded theory (Glaser \& Strauss, 1967; Strauss \& Corbin, 1990), a qualitative methodology that aims to uncover and explore the meanings that construct the participants' realities and behaviours that stem from them, was used to guide this study. A theory or model is then developed that explores the basic social processes that are present within these human interactions. The key dimensions of the construct of compassion

This article is protected by copyright. All rights reserved. 
generated the HCP Compassion Model (Shane Sinclair et al., 2018), whereas the aspects of the model describing and illustrating the facilitators and challenges affecting compassion are presented herein (Figure1). Grounded theory is a particularly powerful method for studies where the phenomenon of interest is complex, has not been well described, and is socially constructed. In grounded theory, data collection and analysis occur simultaneously, with analysis driving further data collection. Five members of the research team (SS, TH, SM, $\mathrm{SRB}, \mathrm{KS}$ ) analyzed the data across the three stages of analysis in grounded theory. A detailed account of the methods associated with this study were published in a separate manuscript (Shane Sinclair et al., 2018).

\section{RESULTS}

\section{Category: Challenges to Compassion}

Participants identified a number of challenges that inhibited their ability to be compassionate (Table 5). These included personal challenges which involved egotistic caregiving and individual differences in the requisite qualities involved in providing compassion. A second theme, relational challenges included HCPs' prejudice towards patients, and a lack of patient and family receptivity; and the third theme, systemic challenges, focused on competing system demands and time constraints. While HCPs described a number of factors that affected their ability to be compassionate, most of them also described them as challenges to be overcome versus barriers in the absolute sense of the word. In fact, how one responds to these so-called barriers to compassion, involving challenging patients, circumstances or profound HCPs personal issues, were often cited as the rationale for nominating their exemplary compassionate colleagues (Stage 2). This important reframing of barriers as challenges that could be overcome, was felt to guard against treating

This article is protected by copyright. All rights reserved. 
barriers as insurmountable or excuses for not being compassionate and are adopted in the presentation of results below.

\section{Theme: Personal Challenges}

Participants described internal, personal factors as a major challenge to compassion. Personal challenges included the personal perspectives and attitudes of HCPs, which influenced their ability to be compassionate to patients. In particular, participants identified egotistic caregiving, whereby HCPs overemphasized their role and unique ability in the caregiving process as a significant, though seemingly subtle, challenge to providing compassion. Conversely, participants also identified variability in individual HCPs' innate capacity for compassion and viewing compassion as being weak as additional internal challenges to providing compassion. While participants recognized differing baseline capacities for compassion and the need to challenge assumptions about the topic, they nonetheless felt that compassion was something that could be nurtured and strengthened with practice.

\section{Sub Theme: Egotistic Caregiving}

Participants described perceived self-importance in the provision of care as a significant challenge to providing compassion. Some participants described this phenomenon as having a 'messiah complex' and 'chronic caring', whereby HCPs' ego and need to be perceived as compassionate ironically circumvented the process of compassion itself.

Sometimes we can be barriers ourselves [laughter]. As nurses sometimes we think we know best on what to do or how to control symptoms (Stage 1 Focus Group Participant 33).

So that's a huge barrier to compassion is feeling yourself extremely important and that you're going to make it all better. (Interview Participant 5)

This article is protected by copyright. All rights reserved. 
Although often functioning pervasively under the guise of good intentions, egotistic caregiving caused HCPs to try to 'fix' patient's situations, to over-identify emotionally with the patient's situation, creating a co-dependent relationship and the potential for HCP burnout in the process. As a result, many participants emphasized a self-effacing attitude as a necessary precondition in mitigating this challenge to compassion.

Where I'm the only one who can care for this person because we have such a close relationship, so you're the one who comes riding in on the white horse with the white hat to save the day, because I'm the only one. And so, I think that muddies the road of compassion... Because it becomes less about that person and it's about---it becomes more I feel better because now this person relies on me (Interview Participant 7)

Just forget about everything, about yourself and just focus on the person (Stage 1 Focus Group Participant 20)

\section{Sub-theme: Individual differences in HCPs innate virtues}

Participants described an internal tension that was an inherent challenge in being compassionate, namely, that the ability to provide compassion was somewhat contingent on the innate virtues HCPs possessed— qualities that HCPs felt were antecedents to compassion (Shane Sinclair et al., 2018). Participants believed that while most HCPs were capable of being compassionate, the requisite qualities that served as motivators to compassion were heavily influenced by individual HCP life experience and their personalities. This served as both an individual and collective challenge for HCPs as the continuity of compassionate care experienced by patients could be positively or negatively affected by variance in other team members underlying virtues. While acknowledging that HCPs' capacity for compassion varied based on the innate qualities and life experience of the individual, participants felt that all HCPs could choose to either nourish or stifle it.

I think there's compassion in all of us. I think it's through experiences and how you learn from those experiences or grow from those experiences that can either nourish that compassion or it can stifle it. (Interview Participant 8)

This article is protected by copyright. All rights reserved. 
I believe it's there, it just gets really well hidden sometimes. (Stage 1 Focus Group Participant 26)

\section{Theme: Relational Challenges}

HCPs' intrapersonal issues not only impeded their personal capacity for compassion, but their ability to extend compassion to others, often manifesting in personal prejudices toward certain patients. Prejudice toward patients was not only a by-product of the personal qualities and attitudes that HCPs' brought into the clinical encounter, but were enculturated through the practice setting and other members of the healthcare team. While participants felt that enculturation of stigmatization towards patients, particularly individuals from vulnerable populations, was often perpetuated in an implicit manner within the consult notes of the patient's medical record, it was considered by many participants to be one of the strongest inhibitors of compassion. Conversely, participants also identified that patient and family members' interpersonal attitudes toward HCPs were also relational inhibitors that challenged their ability to be compassionate. In particular, they identified anger, denial, and physical and verbal abuse as being significant challenges.

\section{Sub-theme: 'Conditional Compassion': Stigmatization and prejudice towards patients}

While participants noted that the field of palliative care purports to be non-conditional in provision of care to patients from all backgrounds, in practice, participants felt that patients who were perceived as being non-compliant or did not behave in a manner that was deemed acceptable to HCPs were sometimes less likely to receive care that was compassionate. In particular, patients who were perceived as displaying drug seeking behavior, were homeless, or were 'frequent flyers' of the healthcare system, were felt to be particularly susceptible to prejudice by individuals and healthcare teams. Study participants had no shortage of clinical examples of situations where they witnessed the power of stigmatization as a counter agent of

This article is protected by copyright. All rights reserved. 
compassion and stressed the need to be aware and actively attend these challenges in their individual practice and the broader culture of care.

So someone was accused of taking medications from the home and so there's this big judgment because it was narcotics and it was a large amount of narcotics that wasn't in the home... And so there was all these negatives on this poor guy...it turned out that --- he had indeed sent the stuff back to the pharmacy (Interview Participant 2).

At the hospital I was working at we had a pretty high proportion of homeless patients and patients with addiction and the minute the hospitalization was attributed to something the patient did to themselves or that kind of attitude, that significantly impacted patient care (Stage 1 Focus Group Participant 11).

Sub-theme: 'Conditional Compassion': Perceived Lack of Patient and Family Receptivity to Compassion

Participants in this study also described challenging patients and family relationships as impacting their ability to be compassionate-particularly those who were angry or considered to be 'in denial' of their palliative condition.

Then you got someone that's swearing at you and trying to take a swing at you, so your compassion changes (Stage 1 Focus Group Participant 3)

But I think it depends on where people are at in terms of their acceptance of their illness or their disease progression. Like they just, some people are at a very angry stage and a very unhappy stage and it doesn't matter what you do or say. (Stage 1 Focus Group Participant 4)

A more pervasive, less overt challenge to compassion that HCPs personally

acknowledged was whether patients or family members were receptive to their compassion. While many participants felt that individuals who were receptive and thankful were easy to be compassionate towards, they and other study participants felt that true compassion was non-conditional. As a result, the majority of HCPs felt that these so-called conditions of compassion were an oxymoron and were more of a limitation of the individual's ability to be compassionate than a condition of the concept itself.

This article is protected by copyright. All rights reserved. 
If a client or a family member comes over immediately very demanding I need this and this and this like right now my compassion immediately goes "pffft". But if they, you know I'm just really struggling and can you help me my compassion goes up. (Stage 1 Focus Group Participant 25).

Sometimes they just totally shut us out and no, we don't need your help. Yeah, and that's where I think part of the challenge for compassion (Stage 1 Focus Group Participant 19)

\section{Theme: Systemic Challenges}

An unsupportive healthcare system and a culture of practice that was perceived as lacking in compassion was also identified as a significant challenge in providing compassion, speaking to how issues within the broader healthcare system can impact HCPs' capacity to care in a compassionate manner. In particular, workplaces and systems that did not value compassion or had excessive competing work demands inadvertently diminished HCPs' ability to provide compassionate care.

\section{Sub-theme: Competing System Demands}

Participants felt that HCPs' compassion was often a spontaneous and innate response,

which could nonetheless be stifled by restrictive institutional and practice guidelines or policies, an overly task-oriented approach, and an overly biomedical focus to care.

Participants also described that being short-staffed, having inflexible schedules, and limited

resources stretched their ability to be compassionate as it caused them to focus on completing tasks rather than the person in their care.

We could provide a little bit more compassionate care, but the system doesn't allow for it (Interview Participant 12)

It's how you do that in an institution, it's the setting that you're in because there's so many other things that get in the way of that, that you have all these other obligations and duties and how do you get, how do you have those moments and still get your job done and all the tasks that you have in front of you for that day. That's a struggle for me. (Stage 1 Focus Group Participant 4)

This article is protected by copyright. All rights reserved. 


\section{Sub-theme: Time Constraints}

Some participants mentioned a lack of time to provide compassion due to an excessive workload as another systemic challenge to compassion. According to study participants, forming good relationships with their patients required time as it fostered an understanding of the person and their needs. Nonetheless, while the majority of participants identified a lack of time as a challenge, a number of participants were quick to note that compassion could happen in a moment and therefore was affected by, but not contingent on time.

Time would be the number one factor... If I was told you've got to go and see that person at nine and you've got to be out by ten, then that starts making me feel a little more pressured and a little more---you feel kind of enclosed (Interview Participant 11)

And it's not again that they [healthcare providers] don't have compassion because I think some of them probably have more than me, but with their time constraint they have a bit more of an agenda (Interview Participant 12)

\section{Theme: Maladaptive Responses-Treating challenges to compassion as excuses for not}

\section{being compassionate}

Although HCPs identified the aforementioned challenges to compassion, ironically, they felt that HCPs' responses to these factors were as influential to their ability to provide care in a compassionate manner, as the challenges themselves. Specifically, participants identified so-called 'difficult patients', system issues, and time barriers as overly invoked excuses for HCPs' lack of compassion. In contrast, most participants felt that compassion could be expressed in the briefest clinical interaction, toward the most challenging patient and within the most demanding healthcare system.

I think it's about quality, not quantity. Yeah, you can be compassionate in a short time. (Stage 1 Focus Group Participant 19)

I wonder though, it's almost a perception you know like people think that they have to have lots of time to be compassionate but even if you're in a

This article is protected by copyright. All rights reserved. 
hurry, even if there, you just have 5 minutes with a person, you can still be compassionate (Stage 1 Focus Group Participant 18)

I think in my mind there is no challenging patient. I might have created the challenge. I thinks that's the common theme I think. To me I think that's the best patient I could work with. (Stage 1 Focus Group Participant 9)

If I look back there's very few circumstances where you cannot not find a way to be compassionate to people (Interview Participant 12)

While the topic of compassion fatigue was mentioned sparingly in discussing barriers to compassion, on the few occasions that it was mentioned, it was met with a similar level of cynicism on the part of participants. Participants were averse to compassion fatigue for two reasons: they felt it suggested that the ability to be compassionate was contingent on HCPs first feeling compassion; and they felt it implied a causal relationship between expressing compassion and burnout. Participants discomfort in viewing compassion as contingent on the affective state of HCPs or treating it as a feeling in its own right, was based on their belief that compassion was rooted in virtues which they felt were more non-conditional and equanimous than feelings. Other participants acknowledged that their affective state and occupational stressors did impede their ability to be compassionate, however they did not feel that that they were specific barriers to compassion, but were general barriers that equally impacted other occupational issues (e.g. professionalism, attentiveness, decision-making, problem-solving, wellbeing, etc.).

There's this thing called compassion fatigue and I actually have a feeling that compassion fatigue isn't actually compassion... So if you're having to work at it, then you get tired and the fatigue isn't exactly in feeling compassion. It's battling all the things that are prior to the compassion and dealing with all the barriers that you have to it...I don't think it's so much about compassion fatigue, but I think it is about fatigue (Interview Participant 5)

I don't know that it [compassion fatigue] challenges my compassion as much as it challenges other things like my sense of doing the right thing or feeling like I'm making a difference or job satisfaction more than it challenges my ability to be compassionate. (Interview Participant 9)

This article is protected by copyright. All rights reserved. 
If we only act compassionate when we're feeling compassionate then we're probably in the wrong business. (Stage 1 Focus Group Participant 24)

\section{Category: Facilitators to Compassion}

In this study, participants shared a number of factors that could facilitate and foster compassion, counteracting challenges to compassion in the process (Table 5). Participants felt that while a supportive system, colleagues, and a compassionate practice setting sustained and increased their ability to be compassionate, many of the associated facilitators resided within HCPs themselves. In addition, expressions of gratitude from patients and their families, and establishing positive and meaningful relational connections served as facilitators of HCPs' compassion.

\section{Theme: Personal Facilitators}

Participants described personal practices that helped cultivate and sustain their ability to provide compassion to patients and families, namely self-care, contemplative practices, and personal experiences of suffering. While the effect of these personal practices extended to their clinical practice, they largely occurred outside of the workplace, suggesting that compassion traverses personal and professional boundaries, requiring self-motivation and personal practice.

\section{Sub-Theme: Self-Care}

The majority of study participants emphasized the importance of self-care as a key facilitator of compassion. Within the demanding context of healthcare, especially in healthcare settings where HCPs where exposed to suffering, the need to take care of oneself was imperative to providing optimal compassion to others. Self-care, self-awareness, and self-reflection were felt to maintain HCPs' physical, emotional, mental, social and spiritual 
wellbeing. This care for the caregiver, in turn was felt to enhance HCPs ability to provide compassion to their patients.

And I think one of the biggest things... to make you compassionate is selfcare. (Stage 1 Focus Group Participant 4).

I think self-care and self-reflections is also part of being compassionate in some way (Stage 1 Focus Group Participant 19)

I think it is important to have a kind of repository where you can put all the suffering and all the pain that you witnessed in that day and give it away in some way, whether it's through prayer or through meditation or maybe just doing something simple like going for a run and resetting the balance by doing something nice for yourself. (Interview Participant 5)

There's lots of people who don't love themselves very much and have a huge capacity to love other people.... I mean I don't always have the best self-esteem or the best self-love, but I think that I provide fairly consistent compassionate care to other people. (Interview Participant 4)

\section{Sub-Theme: Contemplative Practices}

Contemplative or spiritual practices were a second important personal facilitator of

HCP compassion. Whether a formal faith, a meditative practice, a spiritual belief system, or a commitment to self-growth, many participants identified the need to draw personal guidance from a larger source of inspiration as an essential facilitator.

I'm not a religious person but I would say a majority of our staff have a faith of some kind that I see really positively influence their ability to provide compassion. (Stage 1 Focus Group Participant 16).

I think my own mindfulness practice shapes it, just because I keep practicing this... So it hasn't been a linear process; there's been lots of times where I didn't bother with any of that kind of thinking and there were times where I was miserably uncompassionate, but I just kept chipping away at it over the year. (Interview Participant 5)

\section{Sub-theme: Personal Experiences of Suffering}

Participants also identified their own personal experiences of suffering (e.g. grief, personal hardships, and personal experience with illness and death) as a key personal facilitator of compassion. Personal suffering, and particularly grief, was felt to forge a deeper

This article is protected by copyright. All rights reserved. 
understanding of their patients suffering that many participants described as shared-humanity, serving as common ground where compassion could be nourished.

I think [caring for my dying father] allows my compassion to show through in a more genuine way. Not that I wasn't genuine before but just a different layer and a deeper sense of that sincerity and genuineness. (Stage 1 Focus Group Participant 29)

I think it's a prerequisite to be able to be compassionate, because you have seen vulnerability expressed and you know how you want it to be for you and your loved ones in the future (Stage 1 Focus Group Participant 30)

I have a lot of experience with loss and grief personally, so I know and I can understand where these people are coming from. (Interview Participant 13)

Having gone through things here, I can feel a great tenderness and a compassion for the broken pieces I see in someone else. (Interview Participant 6)

\section{Theme: Relational Facilitators}

HCPs described two relational facilitators that sustained and deepened their sense of compassion toward patients and families-expressions of gratitude from patients and families regarding the care they received, and the sense of connection they felt toward their patients and families. Importantly, this acknowledgment served as a point of tension for many HCPs who believed in the unconditional nature of compassion.

\section{Sub-Theme: Expressions of Gratitude \& Positive Feedback for HCP Compassion}

Expressions of gratitude on the part of patients and families seemed to increase HCPs' desire to express compassion to these individuals and seemed to have a snowball effect in their subsequent interactions with these individuals and other patients in their care.

It is easier to be compassionate to a person who is thankful for you, for your work, for what you do. And it's like I was saying, it's more difficult to be compassionate to difficult people who don't see, like where you can't seem to do anything right, nothing satisfies them, there's always something wrong and it's really hard to continue giving that selfless love to that person (Stage 1 Focus Group Participant 14)

This article is protected by copyright. All rights reserved. 
While general expressions of gratitude helped HCPs know that their

compassion was appreciated by the patient, knowing that their compassion also

made a difference or had a meaningful impact for the patient seemed to be equally

important in facilitating further expressions of compassion

Gratitude and thank you cards and mentions in obituaries and all of those things are kind of like the fuel to keep going, at least in my perspective. And the reward to say hey, this made a difference, this compassion meant something and people are responding in whatever way they think will be meaningful to us. (Stage 1 Focus Group Participant 16)

I find that my cup gets filled when I give compassion and when I show compassion and I get a positive response back. (Stage 1 Focus Group Participant 28)

\section{Sub-theme: Sense of Connection}

The majority of HCPs cautioned against compassion being predicated on a preexisting relationship. However, they did nonetheless acknowledge that an established relationship, in which they knew their patients' needs and preferences, allowed their compassion to have a more personal, targeted and impactful effect.

The more connected or the stronger the relationship with the family or the more connection you have with the family, certainly the compassion flows easier. (Interview Participant 12)

I think you can still be compassionate at that level, but I think it's different if you go---there's a deeper level if you can go deeper with people... You need to establish relationship, which is hard to do at a very surface level. (Interview Participant 8)

\section{Theme: Systemic facilitators- Compassionate Organizational and Practice Cultures}

A third facilitator emerging from the data was the need for compassionate healthcare systems and practice settings in sustaining individual HCPs' ability to provide compassion. In particular, HCPs described the importance of having supportive colleagues and at a systems level, leaders that were committed to compassion and organizational values that reflected compassion.

This article is protected by copyright. All rights reserved. 
I couldn't do my job everyday if I didn't have my coworkers to support me (Stage 1 Focus Group Participant 4).

Being a part of your interdisciplinary team, a true collaborative part of it, can make you a better caregiver because have kind of the knowledge and abilities of everyone shared so that you can be more compassionate to a person and understand them better and be in their shoes and walk beside them better (Stage 1 Focus Group Participant 14).

I think sort of organizationally, I think we can help people foster compassion by being compassionate to our employees (Interview Participant 15).

We have the best manager in the world..., her unofficial, or probably official motto is 'It's all about the love'. (Stage 1 Focus Group Participant 8).

\section{Theme: Adaptive Responses- Cultivating Compassion through Intentional Action}

While HCPs identified the personal, relational and the workplace culture as facilitators, most cautioned against treating these as prerequisites to compassion. In fact, participants felt that one of the greatest facilitators, regardless of the existence of personal, relational and system facilitators, was intentional acts of compassion. In doing so, participants described incidences where either they didn't feel compassionate toward an individual or they were met with resistance on the part of patients that was overcome through a compassionate act on the part of HCPs, thus serving as an adaptive response in those challenging situations. Intentional action could take the form of tangible acts such as getting a warm blanket for a frail patient, repositioning a patient, resolving a family complaint, or advocating on the patients' behalf, which in turn allowed HCPs' compassion to begin to flow more freely.

To be perfectly honest I mean, yeah, I mean it's not my father, it's not my mother, it's not my daughter or son, but yet the minute you're in the door there's just something there that just flips on this little compassion switch and it's just really cool to know that even though you're a perfect stranger you can display compassion (Stage 1 Focus Group Participant 25)

Like I just feel myself going I'm trying to help you and you're not working with me. Work with me you know. But when I find them kind of going

This article is protected by copyright. All rights reserved. 
against me, then I find myself saying 'take a step back, take a deep breath and just kind of listen and hear what they're saying and hear what they want. (Interview Participant 11)

There could have been things that could really get in the way; there was things that got in the way because the group home wouldn't take her back. But I kept working with the mum to find a way. (Interview Participant 2)

\section{DISCUSSION}

Our previously published patient (S. Sinclair, McClement, et al., 2016) and HCP model of compassion (Shane Sinclair et al., 2018), along with other studies, focused on the nature of compassion (Armstrong, Parsons, \& Barker, 2000; Bray et al., 2014; Graber \& Mitcham, 2004; Horsburgh \& Ross, 2013; Kvangarsnes, Torheim, Hole, \& Crawford, 2013; Lloyd \& Carson, 2011; Lown et al., 2011; Papadopoulos et al., 2017; Papadopoulos et al., 2016; Skaff, Toumey, Rapp, \& Fahringer, 2003; Van Der Cingel, 2011; Vivino et al., 2009; Way \& Tracy, 2012); identified that a comprehensive understanding of the facilitators and barriers to compassion derived from a robust sample of HCPs was lacking. Thus, this study aimed to identify a comprehensive understanding of the barriers and facilitators of compassion and their impact on the flow of compassion within the HCP compassion model (Figure 1). Two categories along with associated themes and sub-themes emerged from the data (Table 5). Participants identified four themes and six sub-themes that challenged their ability to provide compassion (Table 5). One of the unanticipated findings from this study was participants' aversion toward associating the language of barriers to compassion as they felt it applied an absolute term to the non-conditional and adaptive nature or compassion. They did however recognize that the reality of clinical practice and their own human condition introduced challenges to compassion—challenges that HCPs could nonetheless, adaptively overcome.

This article is protected by copyright. All rights reserved. 
The greatest challenges to compassion identified within this study were HCPs' personal challenges, with relational and systemic challenges taking a secondary role. Personal challenges seemed to have a particularly detrimental impact on compassion because they impeded the development and expression of virtues - the initial motivators of compassion, thereby effecting the flow of compassion in all subsequent domains within the HCP compassion model (Figure 1). Egotistic caregiving was one of the most significant personal challenges identified by participants, as it shifted the selfless and person-orientated focus of compassion in order to meet the needs of the HCP. This elevation of the status of the HCP in compassion was also felt to negatively affect HCPs by leading them to overly identify with the emotional state of their patients and trying to fix their situation. Conversely, HCPs emphasized the necessity of adopting a sense of selflessness as a key facilitator to compassion, counteracting their ego and protecting them from over extending themselves. Participants emphasized that a selfless attitude was an important facilitator of compassion, suggesting that the relation of the self to compassion, including the concept of selfcompassion, may need to be reconceptualized (S. Sinclair, Kondejewski, et al., 2017). This finding is aligned with previous studies that described compassion as a selfless attribute of HCPs that aimed to recognize and ameliorate patient's suffering through relational understanding and action (Bray et al., 2014; Crawford, Gilbert, Gilbert, Gale, \& Harvey, 2013; Horsburgh \& Ross, 2013; Schantz, 2007; S. Sinclair, Kondejewski, et al., 2017; S. Sinclair, McClement, et al., 2016; S. Sinclair, Norris, et al., 2016; Vivino et al., 2009; Way \& Tracy, 2012). The other-orientated focus of compassion placed the self in a subordinate position, serving as a conduit through which compassion could flow, in order to benefit the patient.

This article is protected by copyright. All rights reserved. 
Selflessness has also been previously identified as an essential factor for a strong and successful client-therapist relationship and positive patient experience (Aguilar, Stupans, Scutter, \& King, 2012). Selfless attributes such as virtues of humility, kindness and benevolence have been previously identified by both patients and HCPs as motivators of compassion (Shane Sinclair, 2018; S. Sinclair, McClement, et al., 2016). Cumulatively, while these results suggest that compassion ultimately is about the patient and not HCPs; the self can nonetheless be both a significant inhibitor and facilitator of compassion to others. The essential, albeit subordinate role of the self, was further underscored by participants in identifying self-care, self-reflection, self-awareness, and contemplative practices as further facilitators to compassion and effective caregiving in general. Contemplative practices such as a personal faith, meditation and prayer seemed to provide a framework for selfunderstanding, making sense of suffering, cultivating virtues, and serving as a source of wisdom on the topic of compassion specifically.

External factors such as difficult patients and families, lack of time, and a challenging work environment were considered challenges to compassion by HCPs. However, how HCPs chose to adapt to these factors was an equally, and perhaps more powerful mediator of compassion. Thus, while the flow of compassion could be upregulated or downregulated as it traversed through each of the junctures of personal, relational and systemic facilitators and challenges, most HCPs believed that they could still respond to these factors in an adaptive or maladaptive manner (Figure 1). Compassion fatigue, an often-cited challenge to compassion in the healthcare literature, has been described as "a cost of caring" for others who are in significant physical and emotional distress, which can result in physical and emotional exhaustion in HCPs, thereby diminishing their ability to provide compassion (Figley, 1995; Mathieu, 2007; S. Sinclair, Raffin-Bouchal, et al., 2017). In contrast to the compassion fatigue literature which infers a causal relationship between compassion and occupational 
stress or vice versa, participants in our study did not identify compassion fatigue as a challenge to compassion and were largely resistant to attributing fatigue as a specific inhibitor to compassion. This clinical evidence from frontline HCPs directly engaged in providing compassionate care, supports a recent critical appraisal of the compassion fatigue literature within healthcare which identified a lack of evidence and significant limitations associated with this evocative concept (S. Sinclair, Raffin-Bouchal, et al., 2017). In fact, several participants in this study questioned the relationship of compassion fatigue to the overarching concept of compassion, with some participants refuting the concept outright. Participants did nonetheless acknowledge the need for self-care in HCPs, including but not limited to, their ability to be compassionate.

Participants also expressed a belief that providing compassion was not contingent on positive feelings or more specifically, compassion related feelings towards patients or themselves as individuals. Participants identified contemplative practices and self-care as helpful in cultivating virtues which were perceived as the essential building blocks of compassion. Participants believed that compassion involved choice and action, irrespective of the affective state of the responder, echoing previous research on the topic (Barratt, 2017; Gale et al., 2017). The identification of intentional acts of compassion as a way of engendering compassion in situations where HCPs did not necessarily feel compassion towards a patient, was described as an adaptive response that could in turn stimulate the virtues of compassion. This result suggests that although there is a natural flow to compassion--motivated by the virtues of HCPs, triggering a response that involves seeking to understand the person, relational communicating and attending to their needs, in practice it is not necessarily a sequential process. This finding also challenges the notion of compassionsatisfaction, "the pleasure HCPs derive from being able to do their work" (Stamm, 2010), as a key facilitator of compassion within the healthcare literature. While study participants

This article is protected by copyright. All rights reserved. 
acknowledged that feelings derived from helping others and expressions of gratitude can indirectly promote ongoing compassion to others, the results of this study suggest that a strict cause-effect relationship between HCP satisfaction with caregiving and increased compassion is limited (Sacco, Ciurzynski, Harvey, \& Ingersoll, 2015).

Results of this study also confirm and extend previous research investigating how relational issues between HCPs and their patients can inhibit HCP compassion (A. T. Fernando \& N. S. Consedine, 2014; Jones et al., 2016; Vivino et al., 2009). While participants in our study described difficult, aggressive, or demanding patients and families as impacting their ability to be compassionate, they also felt that compassion was highly adaptable in these situations where they faced resistance from individuals or difficult situations. HCPs identified their own personal experiences of suffering, loss, grief, and of receiving compassion as additional resources when encountering relational challenges to compassion, as they provided a means for understanding the individuals' distress and their reaction to their situation.

Our study also extends the existing literature on workplace and systems inhibitors of compassion (Barron et al., 2017; Bray et al., 2014; Brown et al., 2014; Curtis et al., 2012; Dewar \& Mackay, 2010; Dewar \& Nolan, 2013; Fernando \& Consedine, 2017; Horsburgh \& Ross, 2013; Jones et al., 2016; Lown et al., 2011; Nolan, 2016; Smith et al., 2014), recognizing that while compassion is inherently relational, these relationships occur and are affected by the broader culture of care that they reside in. Our results stress the critical and often overlooked role that practice cultures, organizations and healthcare systems play in maintaining and enhancing compassion in healthcare. Frontline HCPs and their nominated exemplary compassionate care peers felt that compassion was a collective effort that was fostered through environments characterized by supportive colleagues, positive feedback, a person-centered and a bio/psycho/spiritual approach to care. They also profiled the positive

This article is protected by copyright. All rights reserved. 
effect of leaders who proactively supported and modeled compassionate care at both the bedside and at a systems level. Currently, healthcare systems and a healthcare career seem to have a detrimental effect on an individual's innate compassion (A. T. Fernando, 3rd \& N. S. Consedine, 2014). Both our results and other emerging research suggest that by developing a culture of compassion within practice settings and the larger healthcare system, this troubling trend may be reversed. This holds the potential to enhance job satisfaction, reduce workrelated distress and improve patient care in the process (Figley, 1995; Mathieu, 2007; Stamm, 2010).

The articulation and illustration (Figure 1) of specific challenges and facilitators to compassion through the eyes of HCPs, builds on our previous research describing what constitutes compassionate health care according to both patients and HCPs (Shane Sinclair et al., 2018; S. Sinclair, McClement, et al., 2016). Although this empirical foundation helps to delineate the landscape of major elements of compassionate health care delivery, it raises many more questions including whether it is possible to intervene, to purposely reduce challenges to compassionate healthcare and to enhance facilitators. Considerable work has been undertaken in the field of Implementation Science to understand barriers and facilitators of change, particularly as related to HCP behaviors. This body of work arose out of awareness over 20 years ago that wide dissemination of evidence based clinical practice guidelines which articulate best clinical practices, often are ineffective in changing HCPs' behavior. Subsequent work has been undertaken to identify, understand, and intervene to reduce barriers and enhance facilitators to the desired change in behavior. To that end, there are numerous methodological approaches which have been developed and tested. One widely cited framework is Michie's Theoretical Domains Framework (Cane, O'Connor, \& Michie, 2012; Michie et al., 2005). The model has gained substantial traction in the health systems research community as related to understanding and then bringing about change in HCP 
behavior (Graham et al., 2006). Thus, in having identified the specific challenges and facilitators of compassion in this qualitative study, a logical next step would be to apply these findings through an Implementation Science framework to determine how this evidence can be best implemented in practice and what steps are needed to support improvement in the delivery of compassionate care by HCPs.

\section{CONCLUSIONS}

While this study, along with recent high-profile healthcare reviews, suggest that compassion in our contemporary healthcare system is challenged, study participants did not view these challenges as insurmountable barriers but rather opportunities that needed to be addressed at a system, relational and individual level. The study results indicate the journey to improving compassion is a collective responsibility. HCPs have indicated that treating these challenges as barriers or excuses to providing compassionate care can circumvent these efforts and can unjustly project blame when compassion is lacking to individual HCPs, management, or 'the system'. We suggest that the most effective starting point for improving compassion in healthcare, regardless of one's role or responsibility, is to begin by looking at ourselves and how we can each improve compassion within our sphere of influence whether that be at the bedside or the boardroom.

\section{RELEVANCE TO CLINICAL PRACTICE}

Our study provides an in-depth understanding of the challenges and facilitators of compassion directly from the perspectives of HCPs, extending the current knowledge base which either focussed on specific facets, was anecdotal or did not account for the clinical realities that HCPs face each day. While our study confirmed the role that external factors such as the role that the healthcare system plays in providing compassion, participants in this

This article is protected by copyright. All rights reserved. 
study identified personal challenges as additional significant inhibitors. A further insight of this study was the tension and aversion that HCPs expressed in attributing challenges to compassion as barriers. This suggests that while compassion is not immune to personal, relational and system challenges; it is also in essence dynamic, versatile and adaptable to the situation and individuals involved during clinical practice.

\section{LIMITATIONS}

While participants worked in a variety of practice settings, all of them worked within palliative care and therefore the generalizability of our results may not be transferable to other areas of healthcare. Although we suspect that our results will resonate with HCPs working in other areas of healthcare, we postulate that the facilitators and challenges to compassion identified in this study will likely need to be contextualized to individual practice settings. There is also the possibility that our sample was not representative of palliative care providers as our sampling techniques may have accrued a homogeneous group while excluding individuals who were jaded to the topic or had left palliative care. Likewise, our sample consisted of participants who were predominately females (86\%), nurses $(45.6 \%)$ or physicians (22.8) and from a Christian faith background (52.6\%); the study cohort was under representative of males, other healthcare professions and other faith backgrounds. As a result, our findings need to be replicated in other palliative care settings, healthcare fields and cultures to determine the universality and variance of the facilitators and challenges that were identified.

This article is protected by copyright. All rights reserved. 


\section{What does this paper contribute to the wider global clinical community?}

- Provides an in-depth understanding of the challenges and facilitators of compassion directly from the perspectives of frontline healthcare providers.

- Provides a blueprint for optimizing compassion on a personal, relational and system level

- Provides a foundation to researchers and educators develop curricula and future research

\section{REFERENCES}

Aguilar, A., Stupans, I., Scutter, S., \& King, S. (2012). Exploring professionalism: the professional values of Australian occupational therapists. Aust Occup Ther J, 59(3), 209-217. doi:10.1111/j.1440-1630.2012.00996.x

Armstrong, A. E., Parsons, S., \& Barker, P. J. (2000). An inquiry into moral virtues, especially compassion, in psychiatric nurses: findings from a Delphi study. $J$ Psychiatr Ment Health Nurs, 7(4), 297-305.

Barratt, C. (2017). Exploring how mindfulness and self-compassion can enhance compassionate care. Nurs Stand, 31(21), 55-63. doi:10.7748/ns.2017.e10671

Barron, K., Sloan, G., \& Deery, R. (2017). Community mental health nurses' understanding of compassion: a naturalistic, interpretative approach. J Psychiatr Ment Health Nurs. doi:10.1111/jpm.12379

Bray, L., O'Brien, M. R., Kirton, J., Zubairu, K., \& Christiansen, A. (2014). The role of professional education in developing compassionate practitioners: A mixed methods study exploring the perceptions xof health professionals and pre-registration students. Nurse Educ Today, 34. doi:10.1016/j.nedt.2013.06.017

Brown, B., Crawford, P., Gilbert, P., Gilbert, J., \& Gale, C. (2014). Practical compassions: Repertoires of practice and compassion talk in acute mental healthcare. Sociol Health Illn, 36. doi:10.1111/1467-9566.12065

Cane, J., O'Connor, D., \& Michie, S. (2012). Validation of the theoretical domains framework for use in behaviour change and implementation research. Implement Sci, 7, 37. doi:10.1186/1748-5908-7-37

Crawford, P., Gilbert, P., Gilbert, J., Gale, C., \& Harvey, K. (2013). The language of compassion in acute mental health care. Qual Health Res, 23. doi:10.1177/1049732313482190

Curtis, K., Horton, K., \& Smith, P. (2012). Student nurse socialisation in compassionate practice: A Grounded Theory study. Nurse Educ Today, 32. doi:10.1016/j.nedt.2012.04.012

Dewar, B., \& Mackay, R. (2010). Appreciating and developing compassionate care in an acute hospital setting caring for older people. Int J Older People Nurs, 5. doi:10.1111/j.1748-3743.2010.00251.x

This article is protected by copyright. All rights reserved. 
Dewar, B., \& Nolan, M. (2013). Caring about caring: Developing a model to implement compassionate relationship centred care in an older people care setting. Int J Nurs Stud, 50. doi:10.1016/j.ijnurstu.2013.01.008

Fernando, A. T., 3rd, \& Consedine, N. S. (2014). Development and initial psychometric properties of the Barriers to Physician Compassion questionnaire. Postgrad Med J, 90(1065), 388-395. doi:10.1136/postgradmedj-2013-132127

Fernando, A. T., 3rd, \& Consedine, N. S. (2017). Barriers to Medical Compassion as a Function of Experience and Specialization: Psychiatry, Pediatrics, Internal Medicine, Surgery, and General Practice. J Pain Symptom Manage. doi:10.1016/j.jpainsymman.2016.12.324

Fernando, A. T., \& Consedine, N. S. (2014). Development and initial psychometric properties of the Barriers to Physician Compassion questionnaire. Postgrad Med J, 90. doi:10.1136/postgradmedj-2013-132127

Figley, C. R. (1995). Compassion fatigue: Coping with secondary traumatic stress disorder in those who treat the traumatized. Brunner/Mazel psychological stress series, No. 23. Philadelphia, PA: Brunner/Mazel; US.

Frampton, S. B., Guastello, S., \& Lepore, M. (2013). Compassion as the foundation of patient-centered care: the importance of compassion in action. J Comp Eff Res, 2(5), 443-455. doi:10.2217/cer.13.54

Gale, C., Schroder, T., \& Gilbert, P. (2017). 'Do You Practice What You Preach?' A Qualitative Exploration of Therapists' Personal Practice of Compassion Focused Therapy. Clin Psychol Psychother, 24(1), 171-185. doi:10.1002/cpp.1993

Glaser, B. G., \& Strauss, A. L. (1967). The discovery of grounded theory; strategies for qualitative research. Chicago,: Aldine Pub. Co.

Graber, D. R., \& Mitcham, M. D. (2004). Compassionate clinicians: take patient care beyond the ordinary. Holist Nurs Pract, 18(2), 87-94.

Graham, I. D., Logan, J., Harrison, M. B., Straus, S. E., Tetroe, J., Caswell, W., \& Robinson, N. (2006). Lost in knowledge translation: time for a map? J Contin Educ Health Prof, 26(1), 13-24. doi:10.1002/chp.47

Hem, M. H., \& Heggen, K. (2004). Is compassion essential to nursing practice? Contemp Nurse, 17. doi:10.5172/conu.17.1-2.19

Horsburgh, D., \& Ross, J. (2013). Care and compassion: the experiences of newly qualified staff nurses. J Clin Nurs, 22(7-8), 1124-1132. doi:10.1111/jocn.12141

Jones, J., Winch, S., Strube, P., Mitchell, M., \& Henderson, A. (2016). Delivering compassionate care in intensive care units: nurses' perceptions of enablers and barriers. J Adv Nurs, 72(12), 3137-3146. doi:10.1111/jan.13064

Kvangarsnes, M., Torheim, H., Hole, T., \& Crawford, P. (2013). Nurses' perspectives on compassionate care for patients with exacerbated chronic obstructive pulmonary disease. J Allergy Ther, 4. doi:10.4172/2155-6121.1000158

Lloyd, M., \& Carson, A. (2011). Making compassion count: Equal recognition and authentic involvement in mental health care. Int J Consumer Stud, 35. doi:10.1111/j.14706431.2011.01018.x

Lown, B. A., Rosen, J., \& Marttila, J. (2011). An agenda for improving compassionate care: A survey shows about half of patients say such care is missing. Health Aff (Millwood), 30. doi:10.1377/hlthaff.2011.0539

Mathieu, F. (2007). Running on Empty: Compassion Fatigue in Health Professionals (Vol. 4).

Michie, S., Johnston, M., Abraham, C., Lawton, R., Parker, D., Walker, A., \& Psychological Theory, G. (2005). Making psychological theory useful for implementing evidence

This article is protected by copyright. All rights reserved. 
based practice: a consensus approach. Qual Saf Health Care, 14(1), 26-33. doi:10.1136/qshc.2004.011155

Nolan, T. (2016). Compassion, continuity and caring: a new GP's perspective. Br J Gen Pract, 66(643), 93. doi:10.3399/bjgp16X683665

Papadopoulos, I., Taylor, G., Ali, S., Aagard, M., Akman, O., Alpers, L. M., . . Zorba, A. (2017). Exploring Nurses' Meaning and Experiences of Compassion: An International Online Survey Involving 15 Countries. J Transcult Nurs, 28(3), 286-295. doi:10.1177/1043659615624740

Papadopoulos, I., Zorba, A., Koulouglioti, C., Ali, S., Aagard, M., Akman, O., . . . Vasiliou, M. (2016). International study on nurses' views and experiences of compassion. Int Nurs Rev, 63(3), 395-405. doi:10.1111/inr.12298

Roberts, L. W., Warner, T. D., Moutier, C., Geppert, C. M., \& Green Hammond, K. A. (2011). Are doctors who have been ill more compassionate? Attitudes of resident physicians regarding personal health issues and the expression of compassion in clinical care. Psychosomatics, 52. doi:10.1016/j.psym.2011.01.042

Sacco, T. L., Ciurzynski, S. M., Harvey, M. E., \& Ingersoll, G. L. (2015). Compassion Satisfaction and Compassion Fatigue Among Critical Care Nurses. Critical Care Nurse, 35(4), 32-44. doi:10.4037/ccn2015392

Schantz, M. (2007). Compassion: A concept analysis. Nurs Forum, 42. doi:10.1111/j.17446198.2007.00067.x

Shane Sinclair, T. H., Shelley Raffin-Bouchal, Susan McClement, Kelli Stajduhar, Pavneet Singh, Neil A. Hagen, Aynharan Sinnarajah, Harvey Max Chochinov. (2018). What are Healthcare Providers' Understandings and Experiences of Compassion? The Healthcare Compassion Model: A Grounded Theory Study of Healthcare Providers in Canada. BMJ Open (Submitted).

Sinclair, S., Beamer, K., Hack, T. F., McClement, S., Raffin Bouchal, S., Chochinov, H. M., \& Hagen, N. A. (2017). Sympathy, empathy, and compassion: A grounded theory study of palliative care patients' understandings, experiences, and preferences. Palliat Med, 31(5), 437-447. doi:10.1177/0269216316663499

Sinclair, S., Bouchal, S. R., Chochinov, H., Hagen, N., \& McClement, S. (2012). Spiritual care: how to do it. BMJ Support Palliat Care, 2(4), 319-327. doi:10.1136/bmjspcare2011-000191

Sinclair, S., Hack, T. F., Raffin-Bouchal, S., McClement, S., Stajduhar, K., Singh, P., . . Chochinov, H. M. (2018). What are healthcare providers' understandings and experiences of compassion? The healthcare compassion model: a grounded theory study of healthcare providers in Canada. BMJ Open, 8(3). doi:10.1136/bmjopen2017-019701

Sinclair, S., Kondejewski, J., Raffin-Bouchal, S., King-Shier, K. M., \& Singh, P. (2017). Can Self-Compassion Promote Healthcare Provider Well-Being and Compassionate Care to Others? Results of a Systematic Review. Appl Psychol Health Well Being. doi:10.1111/aphw.12086

Sinclair, S., McClement, S., Raffin-Bouchal, S., Hack, T. F., Hagen, N. A., McConnell, S., \& Chochinov, H. M. (2016). Compassion in Health Care: An Empirical Model. J Pain Symptom Manage, 51(2), 193-203. doi:10.1016/j.jpainsymman.2015.10.009

Sinclair, S., Norris, J. M., McConnell, S. J., Chochinov, H. M., Hack, T. F., Hagen, N. A., . . . Bouchal, S. R. (2016). Compassion: a scoping review of the healthcare literature. BMC Palliat Care, 15, 6. doi:10.1186/s12904-016-0080-0

Sinclair, S., Raffin-Bouchal, S., Venturato, L., Mijovic-Kondejewski, J., \& SmithMacDonald, L. (2017). Compassion fatigue: A meta-narrative review of the healthcare literature. Int J Nurs Stud, 69, 9-24. doi:10.1016/j.ijnurstu.2017.01.003

This article is protected by copyright. All rights reserved. 
Sinclair, S., Russell, L. B., Hack, T. F., Kondejewski, J., \& Sawatzky, R. (2016). Measuring Compassion in Healthcare: A Comprehensive and Critical Review. The Patient: Patient Centred Outcomes Research. doi:10.1007/s40271-016-0209-5

Sinclair, S., Torres, M. B., Raffin-Bouchal, S., Hack, T. F., McClement, S., Hagen, N. A., \& Chochinov, H. M. (2016). Compassion training in healthcare: what are patients' perspectives on training healthcare providers? BMC Med Educ, 16, 169. doi:10.1186/s12909-016-0695-0

Skaff, K. O., Toumey, C. P., Rapp, D., \& Fahringer, D. (2003). Measuring compassion in physician assistants. JAAPA, 16.

Smith, S., Gentleman, M., Loads, D., \& Pullin, S. (2014). An exploration of a restorative space: A creative approach to reflection for nurse lecturer's focused on experiences of compassion in the workplace. Nurse Educ Today, 34. doi:10.1016/j.nedt.2014.03.003

Stamm, B. H. (2010). The concise manual for the professional quality of life scale. Retrieved from http://www.proqol.org/uploads/ProQOL_Concise_2ndEd_122010.pdf

Strauss, A. L., \& Corbin, J. M. (1990). Basics of qualitative research : grounded theory procedures and techniques. Newbury Park, Calif.: Sage Publications.

Van Der Cingel, M. (2011). Compassion in care: a qualitative study of older people with a chronic disease and nurses. Nurs Ethics, 18(5), 672-685. doi:10.1177/0969733011403556

Vivino, B. L., Thompson, B. J., Hill, C. E., \& Ladany, N. (2009). Compassion in psychotherapy: The perspective of therapists nominated as compassionate. Psychotherapy Research, 19. doi:10.1080/10503300802430681

Way, D., \& Tracy, S. J. (2012). Conceptualizing compassion as recognizing, relating and (re) acting: A qualitative study of compassionate communication at hospice. ComM, 79.

This article is protected by copyright. All rights reserved. 
Table 1: Demographic Information for 57 participants

Demographic Information (Numbers expressed as percentages and (n)).

\begin{tabular}{ll}
\hline Mean Age (Years) & 48.6 \\
\hline Men & $14(8)$ \\
\hline Women & $86(49)$ \\
\hline
\end{tabular}

Mean number of years in palliative care (range) 11.8

Employment Status*

Full-time

Part-time

$57.8(33)$

Casual

$33.3(19)$

$7.0(4)$

\section{Profession}

Registered Nurse $45.6(26)$

Physicians 22.8 (13)

Healthcare Aide $7.0(4)$

Spiritual Care Specialist $5.2(3)$

Unit Clerk $3.5(2)$

Occupational Therapist $3.5(2)$

Licensed Practical Nurse $3.5(2)$

Housekeeper $1.7(1)$

Social Worker $1.7(1)$

Psychologist $1.7(1)$

Respiratory Therapist 1.7 (1)

Physiotherapist $1.7(1)$

\begin{tabular}{ll}
\hline Care Setting** & \\
\hline Home Care & $29.8(17)$ \\
\hline Hospice & $26.3(15)$ \\
\hline Hospital Dedicated Palliative Care Unit & $21.0(12)$ \\
\hline Hospital Palliative Care Consult Service & $14.0(8)$ \\
\hline Palliative Care Administrator & $7.0(4)$ \\
\hline Outpatient Oncology Palliative Care Consult Service & $5.2(3)$ \\
\hline Rural Palliative Care Consult Service & $5.2(3)$ \\
\hline Other & $1.7(1)$ \\
\hline & \\
\hline Religious Affiliation* & $52.6(30)$ \\
\hline Christian & $7.0(4)$ \\
\hline Buddhist & $3.5(2)$ \\
\hline Jewish & $1.7(1)$ \\
\hline Muslim & $1.7(1)$ \\
\hline Hindu & $31.5(18)$ \\
\hline None & \\
\hline & \\
\hline Religious and Spiritual Status* & $33.3(19)$ \\
\hline Spiritual and Religious & $56.1(32)$ \\
\hline Spiritual but not Religious & $8.7(5)$ \\
\hline None & \\
\hline
\end{tabular}

* The total for these categories is less then than $100 \%$ due to non-responses by participants

**The total for these categories is more then than $100 \%$ due to some participants working in multiple care settings

This article is protected by copyright. All rights reserved. 


\section{Table 2: Stage 1 Focus Group Guiding Questions}

1. Based on your professional and personal experience, what does compassion mean to you?

2. Can you give me an example of when you felt you provided or witnessed care that was compassionate? [What do you feel were the key aspects of these interactions?]

3. What do you feel are the major influencers of compassionate care in your practice?

4. What do you feel inhibits your ability to provide compassionate care?

5. Do you think patients and/or family members influence the provision of compassionate care? [How or how not?], [If yes, what characteristics of patients and/or families, do you feel facilitate or inhibit compassionate care?]

6. What advice would you give other healthcare providers on providing compassionate care?

7. Do you think we can train people to be compassionate? [If so, how]?

8. What do you feel inhibits your ability to provide compassionate care?

9. Based on your experience what role, if any, do you feel compassion has in alleviating end of life distress? [What happens when compassionate care is lacking?]

10. What impact does providing compassionate care have on your personally and professionally?

11. Is there anything related to compassion that we have no talked about today that you think is important or were hoping to talk about?

This article is protected by copyright. All rights reserved. 


\section{Table 3: Stage 2 Interview Guiding Questions}

1. You have been identified by your peers as possessing great skill in providing compassionate care. What do you feel might be some of the reasons for this recognition? [Why do you think others identify you as a compassionate healthcare provider]?

2. In your own terms, how would you define compassion? [What does compassion mean to you?]

3. How did you become a compassionate caregiver? [What beliefs, situations, individuals and/or life experiences in your life and practice do you feel have informed your understanding and provision of compassionate care? Have you always been that way? [Were you always like that? How did you learn it? Can it be learned?]

4. If you reflect back on your current position, can you walk me through the best example of when you provided compassionate care? [What constitutes compassionate care in an admin/phone triage role? Please guide me through the process of this encounter in a sequential fashion, highlighting the key components of this interaction from the initial approach to the consequences of this interaction?]

5. Based on your professional and personal experiences, what shapes your compassionate care?

6. If you were responsible for training students in compassionate care, how would you go about it? [What would you teach them?]

7. Is there anything that gets in the way of your ability to provide compassionate care?

8. How do patients and/or families influence how your ability to provide compassionate care? [What characteristics of patients and/or families, do you feel facilitate or inhibit compassionate care?]

9. A number of participants have identified the healthcare system as being a significant factor in delivering compassionate care? From your perspective, how does/can the healthcare system facilitate or inhibit compassionate care?

10. In light of the things you've just identified as facilitators and barriers, what suggestions would you have for enhancing compassion at a systems level? [Where and what would you focus your efforts on in order to enhance compassion at a systems level]?

11. From what you've told me so far, it sounds like compassionate care is important. So what happens [to patients, families or HCPs] when compassionate care is lacking?

12. What impact does providing compassionate care have on you personally and professionally?

This article is protected by copyright. All rights reserved. 
13. Our focus group participants, previous studies and review of the literature have reported how critical and fundamental compassion is to providing quality patient care, but we also know that compassionate care varies. So given all that we know about the importance of compassionate care, why aren't healthcare providers more compassionate?

14. Before we end, given all we've talked about, I just want to revisit one of the first questions I asked, which is how do you personally define compassion? [In light of our discussion, what does compassion mean to you?]

15. Is there anything related to compassion that we have not talked about today that you think is important or were hoping to talk about?

This article is protected by copyright. All rights reserved. 


\section{Table 4: Stage 3 Focus Group Questions}

1. Does the healthcare provider model of compassion make sense to you? [Does it resonate with you]? [Why or Why not]?

2. Do you feel there is anything missing from the model?

3. How do you feel this model might be relevant to you and your work?

4. How do you suggest the model might be integrated into healthcare practice and education?

5. Is there anything related to the model that we have not talked about today that you think is important or were hoping to talk about?

This article is protected by copyright. All rights reserved. 
Table 5: Challenges and facilitators to providing compassion

\begin{tabular}{|c|c|c|}
\hline Categories & Theme & Sub-Themes \\
\hline \multirow{7}{*}{$\begin{array}{l}\text { Challenges to } \\
\text { compassion }\end{array}$} & \multirow{2}{*}{ Personal challenges } & Egotistic caregiving \\
\hline & & $\begin{array}{c}\text { Individual differences in HCPs innate } \\
\text { virtues }\end{array}$ \\
\hline & \multirow{2}{*}{ Relational challenges } & $\begin{array}{c}\text { Conditional Compassion: } \\
\text { Stigmatization and prejudice towards } \\
\text { patients }\end{array}$ \\
\hline & & $\begin{array}{l}\text { Conditional Compassion: Perceived } \\
\text { lack of patient and family receptivity to } \\
\text { compassion }\end{array}$ \\
\hline & \multirow{2}{*}{ Systemic challenges } & Competing system demands \\
\hline & & Time constraints \\
\hline & $\begin{array}{c}\text { Maladaptive responses: Treating } \\
\text { challenges to compassion as } \\
\text { excuses for not being } \\
\text { compassionate } \\
\end{array}$ & \\
\hline \multirow{7}{*}{$\begin{array}{l}\text { Facilitators to } \\
\text { compassion }\end{array}$} & \multirow{3}{*}{ Personal facilitators } & Self-care \\
\hline & & Contemplative practices \\
\hline & & Personal experiences of suffering \\
\hline & \multirow{2}{*}{ Relational facilitators } & $\begin{array}{l}\text { Expressions of gratitude \& positive } \\
\text { feedback for HCP compassion }\end{array}$ \\
\hline & & Sense of connection \\
\hline & $\begin{array}{l}\text { Systemic facilitators: Compassionate } \\
\text { organizational and practice culture }\end{array}$ & \\
\hline & $\begin{array}{c}\text { Adaptive responses: Cultivating } \\
\text { compassion through intentional } \\
\text { action }\end{array}$ & \\
\hline
\end{tabular}

This article is protected by copyright. All rights reserved. 
Figure 1: Facilitators and Challenges to Healthcare Provider Compassion Model

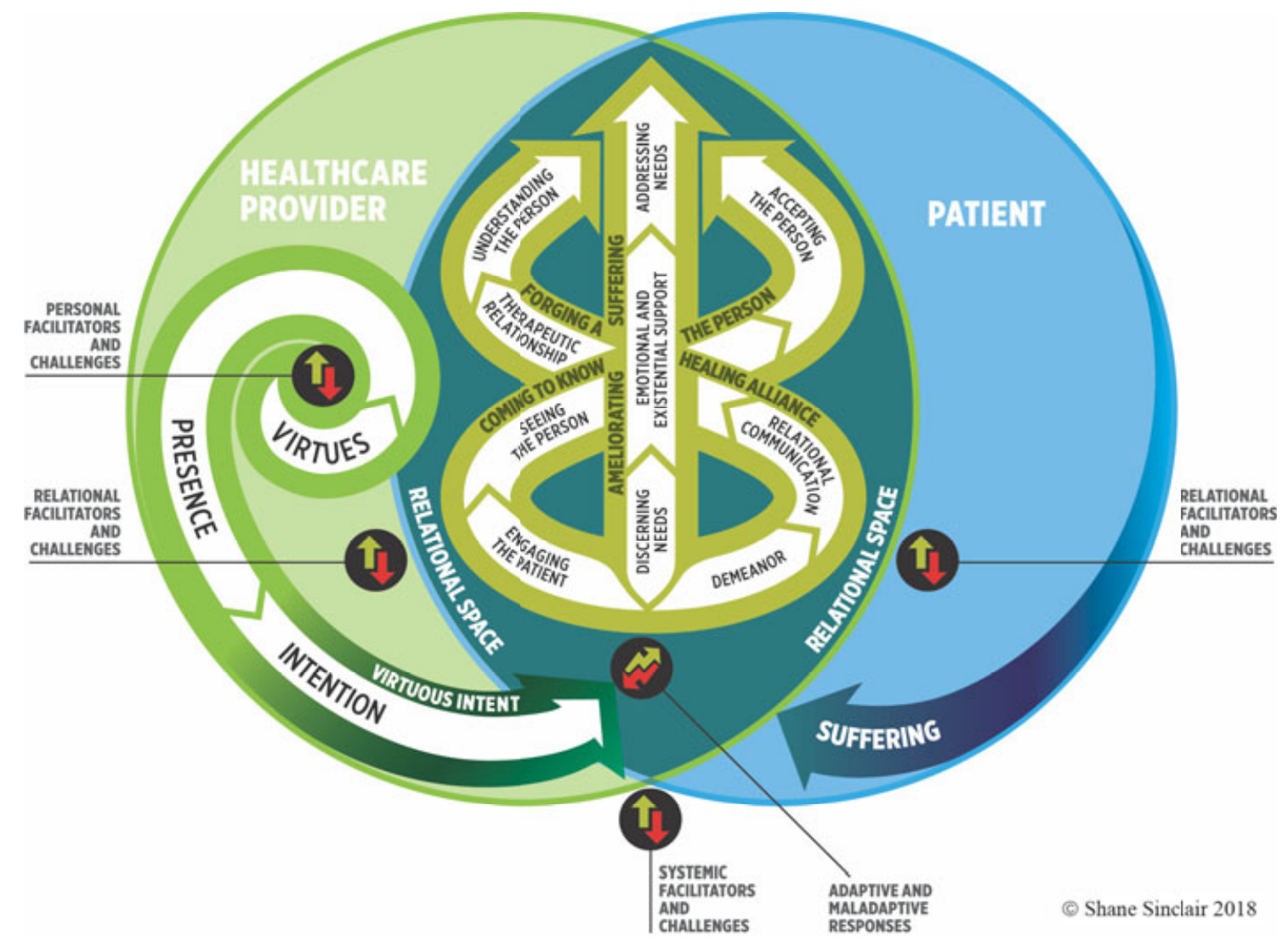

